\title{
Identification of novel circulating angiogenic factors in small bowel angiodysplasia: a further step towards delineating the pathophysiology and defining treatment targets
}

Grainne Holleran ( $\nabla$ grainneholleran@gmail.com )

University of Dublin Trinity College https://orcid.org/0000-0001-8845-4952

Sinead Smith

University of Dublin Trinity College

Deirdre McNamara

University of Dublin Trinity College

Research article

Keywords:

Posted Date: July 30th, 2019

DOl: https://doi.org/10.21203/rs.2.12107/v1

License: (c) (i) This work is licensed under a Creative Commons Attribution 4.0 International License.

Read Full License 


\section{Abstract}

Background: Small bowel angiodysplasia (SBA) accounts for $50 \%$ of small bowel bleeding, with a poor prognosis due to inadequate response to limited treatments. A poor understanding of the pathophysiology of the condition impedes improvements in diagnosis and treatments. We previously identified the Angiopoietin pathway to be associated with SBA, however this may be only one of many angiogenic drivers. Methods: We initially measured relative levels of 55 angiogenic factors in serum of Small Bowel Angiodysplasia (SBA) patients and non-bleeding controls. Quantitative Enzyme-linked Immunosorbent Assay (ELISA) measurements of any significant factors detected were then performed in a larger group of patients and controls. Results: Serum measurements of relative levels of 55 angiogenic factors were performed in seven SBA patients and controls and identified significant differences in five factors between groups- Angiopoietin-1 (Ang1), Angiopoietin-2 (Ang2), Tissue-Inhibitor of Metalloproteinases 1 (TIMP1), Endostatin and Platelet-derived Growth Factor-AA (PDGF-AA). We found no differences in levels of Vascular Endothelial Growth Factor (VEGF). Quantitative serum ELISA measurements of TIMP1, Endostatin and PDGF-AA were performed in 20 SBA patients and controls and showed significantly lower mean levels of TIMP1 and higher levels of Endostatin in SBA patients compared to controls, with no differences in PDGF-AA levels. Conclusions: This study confirms our previous findings, that Ang1 and Ang2 are key factors associated with SBA formation, and that VEGF is unlikely to have a key role in SBA pathophysiology. We have identified two new anti-angiogenic factors likely to be involved in SBA development, TIMP1 and Endostatin. Further assessments of circulating and tissue levels of these factors may advance the discovery of the pathophysiology of SBA and identify therapeutic targets.

\section{Introduction}

Small bowel angiodysplasia (SBA) is quickly becoming recognised as an important cause of small bowel bleeding, accounting for up to $50 \%$ of cases, due to the relatively recent availability of small bowel endoscopy in the form of capsule endoscopy (CE) and enteroscopy ${ }^{1}$. SBA presents clinically with either obscure or overt bleeding, which is generally recurrent despite the use of the best currently available endoscopic and medical therapies ${ }^{2,3}$. Patients with SBA are elderly and have multiple co-morbidities, and the few natural history studies available suggest a poor outcome and reduced quality of life for affected patients ${ }^{4,5}$. The management of SBA could be improved if a better understanding of the pathophysiology of SBA formation was determined, which could identify molecular treatment targets and diagnostic and prognostic biomarkers. Historically angiodysplasia development was thought to be induced by hypoxic changes within the bowel wall, and more recently vascular endothelial growth factor (VEGF) has been implicated in colonic angiodysplasia ${ }^{6-8}$. Angiodysplasias in the small bowel are known to have a worse prognosis than lesions elsewhere, and work we have done previously has failed to determine any association with $\mathrm{VEGF}^{9}$. We have however, identified an association between SBA and the Angiopoietin pathway, namely reduced serum levels of Angiopoietin-1 (Ang1) and elevated levels of Ang2, which we were also able to confirm in tissue samples of SBA. Furthermore, we could prove that these serum 
abnormalities were specific for SBA and not driven by other causes of gastrointestinal bleeding or anaemia, and proposed a model for the use of serum Ang1 and Ang2 levels as a diagnostic aid for $\mathrm{SBA}^{10}$.

However, as angiogenesis is a complex process involving the interaction of a multitude of angiogenic, hypoxic and inflammatory stimuli, the detection of a role of Ang1 and Ang2 in SBA is likely to reflect only a small part of the more complex pathophysiology behind their development. Many studies are now uncovering the complex roles of anti and pro- angiogenic factors, and the concept of factors having dual roles, both inducing and prohibiting angiogenesis is emerging which may occur at certain threshold levels, often influenced by other up or downstream factors ${ }^{11}$. As the field of angiogenesis research is undergoing rapid discoveries, we wanted to ensure that our angiogenesis assessment in SBA formation was as comprehensive as possible. Furthermore, although our initial findings in contrast to earlier studies, did not find an association with VEGF, as one of the most widely studies factors in angiogenesis and with a currently available molecular target, we wanted to gain further clarity of this, and ensure that we were following the correct line of interest in our work with SBA.

\section{Aims And Methods}

- To determine whether the angiopoietin pathway may be only one of several other pathways associated with SBA formation.

- To determine whether up or downstream factors of VEGF, or alternative forms to the one we had previously tested for, were implicated in SBA.

We used a commercially available multiplex angiogenic array kit to initially measure the relative expression of 55 angiogenic factors in the serum of SBA patients and controls. The multiplex Array measured the factors outlined in table 1, and table 2 shows the results of a literature review of the factors and their potential relevance in angiodysplasia, gastrointestinal angiogenesis and any known interactions with VEGF or the Angiopoietins. Significant factors identified were then measured using precise quantitative ELISA measurements.

\section{Human angiogenesis array}

Following informed consent, blood was collected from patients with a definite diagnosis of SBA by CE, and from healthy non-bleeding controls, who had undergone upper and lower endoscopy and CE for a variety of indications but had no history of anaemia, or any potential bleeding source identified by panendoscopy. Samples were centrifuged at 1000rpm for a minimum of 10 minutes and the resultant serum supernatant was then extracted, anonymised, and stored in aliquots at $-80 \mathrm{C}$ for later batch analysis. The human angiogenesis assay (ARY007; R\&D Systems, Minneapolis, MN) was performed on serum samples in duplicate, as per the manufacturer's guidelines. Densitometric values of duplicate samples were averaged and subtracted from the average densitometric value for each negative control to compensate 


\section{Validation of detected factors by ELISA measurement}

Significant differences were detected in five of the 55 angiogenic factors detected by the multi-detection angiogenesis array kit and precise quantitative measurements of three of these factors were then performed using commercially available ELISA kits. Blood samples were collected, processed and stored as in the above step from a larger number of both SBA patients and controls. Data was also collected regarding patient demographics and haemoglobin $(\mathrm{Hb})$ levels. ELISA measurements of the three new factors [Endostatin/Collagen XVIII, TIMP1 (metallopeptidase inhibitor-1) and Platelet derived growth factor (PDGF)-AA] identified by the multiplex angiogenesis array were performed using commercially available ELISA antibody array kits (DNSTO, DTM100, DAAOOB; R\&D systems, Minneapolis, MN). Repeat measurements of Ang1 and Ang2 were not performed. All measurements were performed according to the manufacturer's guidelines. Samples were prepared in duplicate and results were read at $450 \mathrm{~nm}$ absorbance.

\section{Statistical analysis}

Results of all assays and patient demographics were expressed as a mean and/or median and compared between patients and controls using the Student $t$ test, or Mann-Whitney test, as appropriate, with a difference of $<0.05$ considered significant. The presence of confounding variables was determined by using a one way ANOVA test, with a $p$ value of $<0.05$ considered significant. All analyses were performed using SPSS version 20 (SPSS Inc., Chicago, IL).

\section{Results}

\section{Multi-detection angiogenesis array:}

An initial assessment using the 55 antibody array assay was performed in 14 samples including seven SBA patients and seven controls. There were no differences in age or gender in the two groups with mean ages of 60.3 years (range $52-73$ ) and 67.0 years (range $53-79$ ) $[p=0.085]$ and Male: Female gender ratios of $4: 3$ and $5: 2[p=0.306]$ for the control and SBA patient groups respectively. Significantly lower levels of four factors were found in patients with SBA vs controls: Ang-1, PDGF-AA, Endostatin/Collagen VXIII and TIMP1, with a significantly higher level of Ang-2 in SBA patients compared to controls (Table 3). In correlation with our findings in previously published data there were no significant differences in relative expression levels of other factors which had been associated with angiodysplasia in previous publications; including various forms of VEGF, soluble Endoglin (sEnd) and PDGF-AB/BB. 


\section{Serum ELISA measurements of the additional 3 factors identified by the multiplex assay:}

ELISA measurements were performed in 20 patients with SBA and 20 non-bleeding controls. SBA patients were older than controls with mean ages of $72.75(51-93)$ and $45(18-73)$ respectively $(p<0.001,95 \% \mathrm{Cl}$ 18.99 to 36.51$)$, however there was no difference in gender, with females accounting for $40 \%(n=8)$ of the patients with SBA and 55\% $(n=11)$ of the control group $(p=0.355)$. Of the SBA patients, $85 \%(n=17)$ were anaemic, compared to $30 \%(n=6)$ of controls, with a significant difference in the mean Hb levels between the two groups at $10.79 \mathrm{~g} / \mathrm{dL}(9-12.5)$ and $12.59 \mathrm{~g} / \mathrm{dL}(9.6-15.4)$ respectively.

Overall, significant differences were detected in mean levels of Endostatin and TIMP1 between the two groups, however no differences were observed in levels of PDGF-AA (Table 4).

\section{Effect of age, anaemia and gender on levels of each factor:}

One way ANOVA testing was used to determine confounding variables (age $>50$ years, gender and the presence of anaemia) [table 5]. Of these variable factors, only age $>50$ years was found to be a confounding factor affecting Endostatin levels (table 6).

\section{Discussion}

The initial assessment of 55 angiogenic factors identified five factors with significant differences in serum levels between SBA patients and non-bleeding controls; Ang-1, Ang-2, TIMP-1, Endostatin and PDGF-AA. Although the number of patients $(n=7)$ and controls $(n=7)$ in this group were small, we still managed to find statistically significant results which correlated with our previously published findings of alterations in Ang-1 and Ang-2 levels, thereby further strengthening this association. There were no significant differences in the mean expression levels of the remaining 50 angiogenic factors suggesting that the Angiopoietin pathway is likely to be the key pathway in SBA formation. Furthermore, our previously published negative findings of any association of SBA with VEGF, Soluble Endoglin (sEnd), or PDGF-AB/BB, suggested in the literature to be associated with colonic angiodysplasia and a hereditary form of gastrointestinal angiodysplasia, Hereditary Haemorrhagic Telangiectasia, was further supported by negative findings again in this assessment. During a literature review of the specific role of each of the 55 factors tested for by the multiplex kit, we found that approximately half of them had evidence of a significant role in angiogenesis within the gastrointestinal tract, ranging from normal blood vessel development to disordered pro-neoplastic angiogenesis (Table 2). Furthermore, as VEGF is a critical mediator of angiogenesis overall, we performed a review to determine factors which specifically interact with VEGF to exert their angiogenic effect, either at an upstream or downstream level. This identified an even greater proportion of the 55 factors, which we felt made the multiplex assessment a worthwhile and 
relevant assessment tool in our work. The findings of decreased serum levels of Ang-1 and increased serum levels of Ang-2, fit with our previous hypothesis of an alteration in the ratio of Ang-2/Ang-1 levels, with the disordered vessel growth associated with excess Ang-2 winning out over the protective vessel stabilising role of Ang-1. However, the identification of reduced serum levels of three new factors -TIMP-1, Endostatin/Collagen XVIII and PDGF-AA in patients with SBA compared to non-bleeding controls was an interesting new observation.

In the second part of this study we performed a quantitative serum assessment of the factors identified by the multiplex study in SBA patients compared to non-bleeding controls, in a larger cohort which would either confirm or exclude the association detected. As we had already performed quantitative ELISA measurement of Ang-1 and Ang-2 in this cohort, and in a further prospective cohort, which were in fact validated by the multiplex assessment, we elected not to repeat these measurements and performed ELISA assessments of the three new factors only, TIMP-1, Endostatin and PDGF-AA. This assessment confirmed a statistically significant difference in mean serum levels of TIMP-1 and Endostatin between SBA patients and non-bleeding controls, however it rejected any difference in mean levels of PDGF-AA between the two groups. An unexpected finding however, was that although this assessment detected a significant difference in Endostatin between the groups, this difference was contradictory to that of the multiplex study. In fact, the quantitative ELISA assessment determined that patients with SBA had higher mean circulating levels of Endostatin than non-bleeding controls.

Endostatin, an anti-angiogenic factor, is found in only a few organs in humans, of which the small intestine is one. It is has an important role in tumour angiogenesis, and has a currently available recombinant human pharmaceutical form, Endostar, used in lung cancer,and trials of colorectal and gastric cancer. Endostatin directly binds to VEGF receptors 1 and 2 to prevent the downstream VEGF tyrosine-kinase-induced effects, following binding to both receptors. It also causes downregulation of Hypoxia-Inducible Factor (HIF)-1, Matrix Metalloproteinase (MMPs), Fibroblast-Growth Factor (FGF) and binds with a high affinity to certain integrins ${ }^{12-15}$. Elevated circulating levels of Endostatin have been associated with metastatic cancers, Diabetes Mellitus, Chronic Kidney Disease (CKD), Alzheimer's dementia and Coronary Artery Disease ${ }^{16}$. Studies have concluded that the upregulation of Endostatin is likely to be triggered by hypoxia or ischaemia, which would be in keeping with our observation in patients with SBA, a condition which is accepted to be closely related to ischaemia ${ }^{17-19}$. The seemingly paradoxical association of elevated Endostatin (an anti-angiogenic factor) in what are thought to be angiogenic-dependent conditions, has been investigated by several different groups. Early hypotheses suggested that Endostatin is a by-product, or a factor required for the mobilisation of other promoters of angiogenesis, rather than a direct acting factor itself ${ }^{20}$. More recently, a regulatory role in pathological angiogenesis has been attributed to Endostatin, with a disruption in the balance between other pro- and anti-angiogenic factors in favour of uncontrolled angiogenesis ${ }^{21}$.

Another potentially relevant observation is that anti-angiogenic factors have been shown to have longer half-lives than pro-angiogenic factors, which suggests that their detection may not reflect the real-time 
manifestation of their angiogenic effect ${ }^{22}$. This theory may apply to our results and explain the discrepancy between the multiplex and ELISA results, as serum from SBA patients SBA was taken at a single point in time. Although all SBA patients had a history of bleeding, their blood was not always collected at a bleeding episode, and the detection of elevated Endostatin in these patients may reflect the concept of a long half-life rather than over-expression due to its current activity. An interesting observation in patients with CKD was that plasma levels of Endostatin demonstrated a concentrationdependent relationship with disease severity ${ }^{23}$. This may also be relevant to our results, and explain the variability in ranges of Endostatin between patients. We did not classify the severity or burden of disease in SBA patients but it is possible that this may influence levels of Endostatin and would be an interesting future assessment.

There was a suggestion from the multiplex study of a trend towards higher levels of both VEGF and EGVEGF ( $p$ levels of 0.075 and 0.086 respectively), which could in theory become significant if tested in a larger cohort, however our putative ELISA assessment of quantitative serum levels of VEGF in SBA patients and controls allowed us to disregard these trends with reasonable confidence, and focus on the significant results we did obtain. Furthermore, although the majority of the 55 factors assessed interact with VEGF, we determined that only Endostatin appears to be associated with SBA. Interestingly, a few studies have measured the relationship of combined circulating levels of Endostatin and VEGF in angiogenic-related conditions, and found an inverse correlation between the two factors at different time intervals depending on disease activity ${ }^{24,25}$. Hypothetically, this could explain why we have not observed any differences in VEGF levels associated with SBA to date, as elevated levels of Endostatin may reflect a point-in-time decrease in VEGF activity, rather than ruling out any role for VEGF in SBA pathophysiology. Similarly, Ang1 and Ang2 expression is known to be affected by certain threshold levels of VEGF ${ }^{26}$. Our findings to date suggest that VEGF is not the key mediator of angiogenesis in SBA, however it may have an important role in coordinating the expression of other key factors, and future prospective studies including serial VEGF levels may confirm this hypothesis.

TIMP-1 is an anti-angiogenic factor, which binds with high affinity to (MMP) $-9^{27}$. TIMP-1 expression is induced by several pro-inflammatory cytokines, particularly the Interleukins, Tumour Necrosis Factor (TNF)-, and Transforming Growth Factor (TGF)-. TIMP-1 is thought to exhibit two separate modes of action in cell growth and turnover, the first of which is related to its inhibitory action on the MMPs. MMPs cause degradation of the ECM by affecting collagen, elastin and other basement membrane proteins which is a necessary step in coordinated vessel development ${ }^{28}$. TIMPs inhibit this ECM breakdown by preventing their conversion from a latent to active form. Recently, an action of TIMP-1 independent of the MMPs has been discovered, with an ability to potentiate erythroid cells, and to inhibit apoptosis in $B$ cells ${ }^{29,30}$. TIMP-1 has been shown to play a protective role in vascular remodelling, and in an oversimplified hypothesis, a deficiency of TIMP-1 in patients with SBA, may have a role in the development of disordered blood vessel development in the small intestine. The ELISA assessment of serum levels of TIMP-1 confirmed the initial multiplex results, that SBA patients have lower circulating TIMP-1 levels than controls. Many studies have looked at the effects of TIMP-1, particularly in cancer angiogenesis, 
and determined that TIMP-1 can exert either a pro- or anti-angiogenic effect, determined by circulating levels at the time of vessel development ${ }^{31,32}$. Elevated circulating TIMP-1 levels have been reported as a poor prognostic indicator of disease activity in inflammatory conditions, and of a higher burden of disease and reduced likelihood of response to systemic treatments in malignancies ${ }^{33-36}$. A deficiency of circulating TIMP-1 has been associated with cardiovascular disease, however few studies to date have looked at the angiogenic effects of low circulating levels of TIMP-1 to compare our results to ${ }^{37}$. Based on the proposed protective role of TIMP-1 in blood vessel development, it could be hypothesised that the disordered blood vessel development in SBA could be attributed to the loss of this protective factor, although the explanation for our observed association is likely to be far more complex than this. The reported effect of TNF- on TIMP-1 expression is an interesting observation also, as we have previously demonstrated that SBA patients have decreased serum levels of TNF-, and further assessments of the two factors together at different points in disease activity may yield further information into their role in the pathophysiology of SBA formation.

We performed statistical analysis to determine confounding variables which may have influenced levels of Endostatin or TIMP-1, and found that age over 50 years was associated with elevated Endostatin levels irrespective of the presence of SBA, although no confounding variables were detected for TIMP-1 expression. This observation may be explained by the likelihood of increasing co-morbidities of both SBA patients and controls in the presence of advancing age, particularly conditions associated with ischaemia, which occur predominantly in elderly patients. We did not have adequate clinical data to perform a sub-group analysis of co-morbidities to prove this hypothesis, however given that SBA is a condition almost exclusively present in elderly patients, the detection of age as a confounding factor would make its use in clinical practice as a diagnostic or prognostic factor less likely to be of use.

\section{Conclusion}

Several useful conclusions can be drawn from this study which will guide future work in delineating the pathophysiology of SBA formation. Firstly, the multiplex study validated our previously reported detection of an association of SBA with decreased levels of Ang1 and increased levels of Ang2, highlighting the key importance of the Angiopoietin pathway in SBA. Secondly, it outruled an association with VEGF, which reassured us that we were following the correct line of further investigation in the huge pool of angiogenic pathways. Flnally, we have detected two new angiogenic factors with significant associations with SBA, TIMP-1 and Endostatin. Further studies will need to be performed to determine the specific role of each of these factors, and correlation of levels of each of the detected factors and their relationships to each other at specific points in SBA disease activity may be of particular interest in the future.

\section{Abbreviations}

Small Bowel Angiodysplasia (SBA)

Enzyme-linked Immunosorbent Assay (ELISA) 
Angiopoietin-1 (Ang1)

Angiopoietin-2 (Ang2)

Tissue-Inhibitor of Metalloproteinases 1 (TIMP1)

Platelet-derived Growth Factor-AA (PDGF-AA)

Vascular Endothelial Growth Factor (VEGF)

capsule endoscopy (CE)

Soluble Endoglin (sEnd)

Chronic Kidney Disease (CKD)

Hypoxia-Inducible Factor (HIF)

Matrix Metalloproteinase (MMPs)

Fibroblast-Growth Factor (FGF)

Tumour Necrosis Factor (TNF)

Transforming Growth Factor (TGF)

\section{Declarations}

Ethical approval was obtained from the St James's and Tallaght University Hospital Joint Ethics committee. Written informed consent was obtained from all participants prior to their involvement in the study.

Consent for publication-not applicable

The datasets used and/or analysed during the current study are available from the corresponding author on reasonable request.

The authors declare that they have no competing interests.

This study was funded in full by a research grant and scholarship from the Health Research Board of Ireland, grant reference NSAFP-2014-2.

Specific author contributions: GH, SS and DMcN contributed to the design of the research project. Sample collection was performed by GH and DMcN. GH and SS performed the laboratory analysis and data analysis. $\mathrm{GH}$ and $\mathrm{DMcN}$ wrote the paper and all authors have read and approved the final version of the manuscript. 


\section{References}

1. Sami SS , Al-Araji SA , Ragunath K . Review article: gastrointestinal angiodysplasia - pathogenesis, diagnosis and management. Aliment Pharmacol Ther 2014;39:15-34

2. Holleran G, McNamara D. An overview of angiodysplasia: management and patient prospects. Expert Rev Gastroenterol Hepatol. 2018 Sep;12(9)

3. Romagnuolo J, Brock AS, Ranney N. Is endoscopic therapy effective for angioectasia in obscure gastrointestinal bleeding? A systematic review of the literature. J Clin Gastroenterol. 2015;49

4. Holleran G, Hall B, Zgaga L, Breslin N, McNamara D. The natural history of small bowel angiodysplasia. Scand J Gastroenterol. 2016;51(4)

5. Grooteman KV, Matheeuwsen M, van Geenen EJM, Drenth JPH. Decreased health-related quality of life in angiodysplasia patients: A cross-sectional cohort. PLoS One. 2017 May 26;12(5)

6. Boley SJ, Sammartano R, Adams A, et al. On the nature and etiology of vascular ectasias of the colon. Degenerative lesions of aging. Gastroenterology. 1977;72:650-60

7. Baum S, Athanasoulis CA, Waltman AC, Galdabini J, Schapiro RH, Warshaw AL, Ottinger LW. Angiodysplasia of the right colon: a cause of gastrointestinal bleeding. AJR Am J Roentgenol. 1977 Nov;129(5)

8. Junquera F, Saperas E, de Torres I, Vidal MT, Malagelada JR. Increased expression of angiogenic factors in human colonic angiodysplasia. Am J Gastroenterol. 199;94

9. Holleran G, Hall B, O'Regan M, Smith S, McNamara D. Expression of Angiogenic Factors in Patients With Sporadic Small Bowel Angiodysplasia. J Clin Gastroenterol. 2015 Nov-Dec;49(10)

10. Holleran G, Hussey M, Smith S, McNamara D. Assessment of serum angiogenic factors as a diagnostic aid for small bowel angiodysplasia in patients with obscure gastrointestinal bleeding and anaemia. World J Gastrointest Pathophysiol. 2017 Aug 15;8(3)

11. O'Reilly MS, Boehm T, Shing Y, Fukai N, Vasios G, Lane WS, Flynn E, Birkhead JR, Olsen BR,Folkman J. Endostatin: An Endogenous Inhibitor of Angiogenesis and Tumor Growth. Cell. 1997;88

12. Fukumoto $S$, Morifuji M, Katakura $Y$, Nakamura S. Endostatin inhibits lymph node metastasis by a down-regulation of the vascular endothelial growth factor $\mathrm{C}$ expression in tumor cells. Clin Exp Metastasis. 2005;22

13. Li K, Shi M, Qin S. Current Status and Study Progress of Recombinant Human Endostatin in Cancer Treatment. Oncology and Therapy. 2018 Jun 1

14. Kim YM, Hwang S, Kim YM, Pyun BJ, Kim TY, Lee ST, Gho YS, Kwon YG. Endostatin blocks vascular endothelial growth factor-mediated signaling via direct interaction with KDR/Flk-1. J Biol Chem. $2002 ; 277$

15. Wickstrom SA, Alitalo K, Keski-Oja J. Endostatin associates with integrin alpha5beta1 and caveolin-1, and activates Src via a tyrosyl phosphatase-dependent pathway in human endothelial cells. Cancer 
Res. 2002; 62

16. Walia A, Yang JF, Huang YH, Rosenblatt MI, Chang JH, Azar DT. Endostatin's emerging roles in angiogenesis, lymphangiogenesis, disease, and clinical applications. Biochimica et Biophysica Acta (BBA)-General Subjects. 2015 Dec 1;1850(12

17. Deininger MH, Fimmen BA, Thal DR, Schluesener HJ, Meyermann R. Aberrant neuronal and paracellular deposition of endostatin in brains of patients with Alzheimer's disease. J Neurosci. 2002; 22:10621-10626.

18. Sodha NR, Clements RT, Boodhwani M, Xu SH, Laham RJ, Bianchi C, Sellke FW. Endostatin and angiostatin are increased in diabetic patients with coronary artery disease and associated with impaired coronary collateral formation. Am J Physiol Heart Circ Physiol. 2009; 296:H428-H434.

19. Lerman LO, Chade AR. Angiogenesis in the kidney: a new therapeutic target? Curr Opin Nephrol Hypertens. 2009; 18:160-165.

20. O'Reilly MS, Boehm T, Shing Y, Fukai N, Vasios G, Lane WS, Flynn E, Birkhead JR, Olsen BR,Folkman J. Endostatin: An Endogenous Inhibitor of Angiogenesis and Tumor Growth. Cell. 1997;88:277-285.

21. Folkman J. Role of angiogenesis in tumor growth and metastasis. Semin Oncol. 2002; 29:15-18

22. Lee TY, Tjin Tham Sjin RM, Movahedi S, Ahmed B, Pravda EA, Lo KM, Gillies SD, Folkman J, Javaherian K. Linking antibody Fc domain to endostatin significantly improves endostatin halflife and efficacy. Clin Cancer Res. 2008; 14:1487-1493.

23. Chen J, Hamm LL, Kleinpeter MA, Husserl F, Khan IE, Chen CS, Liu Y, Mills KT, He C, Rifai N, Simon EE, He J. Elevated plasma levels of endostatin are associated with chronic kidney disease. Am J Nephrol. 2012; 35:335-340.

24. Wang X, Xu X, Tang L, Song S, Fan C. Interactions between endostatin and vascular endothelial growth factor (VEGF) and inhibition of choroidal neovascularization. International journal of molecular sciences. 2007 Jan 31;8(1):61-9.

25. Hu Y, Hu MM, Shi GL, Han Y, Li BL. Imbalance between vascular endothelial growth factor and endostatin correlates with the prognosis of operable non-small cell lung cancer. European Journal of Surgical Oncology (EJSO). 2014 Sep 1;40(9):1136-42.

26. Hashizume H, Falco' n BL, Kuroda T, et al. Complementary actions of inhibitors of angiopoietin-2 and VEGF on tumor angiogenesis and growth. Cancer Res. 2010;70:2213-2223.

27. Stamenkovic I. Matrix metalloproteinases in tumor invasion and metastasis. InSeminars in cancer biology 2000 Dec 1 (Vol. 10, No. 6, pp. 415-433). Academic Press.

28. Gomez DE, Alonso DF, Yoshiji H, Thorgeirsson UP. Tissue inhibitors of metalloproteinases: structure, regulation and biological functions. Eur J Cell Biol. 1997 Oct;74(2):111-22.

29. Lambert E, Boudot C, Kadri Z, Soula-Rothhut M, Marie-Line SO, Mayeux P, Hornebeck W, Bernard HA, Petitfrere E. Tissue inhibitor of metalloproteinases-1 signalling pathway leading to erythroid cell survival. Biochemical Journal. 2003 Jun 15;372(3):767-74. 
30. Chirco R, Liu XW, Jung KK, Kim HR. Novel functions of TIMPs in cell signaling. Cancer Metastasis Rev. 2006;25(1):99-113.

31. Van Geest RJ, Klaassen I, Lesnik-Oberstein SY, Tan HS, Mura M, Goldschmeding R, et al. Vitreous TIMP-1 levels associate with neovascularization and TGF-beta2 levels but not with fibrosis in the clinical course of proliferative diabetic retinopathy. Journal of cell communication and signaling. 2013;7(1):1-9.

32. Ikenaka Y, Yoshiji H, Kuriyama S, Yoshii J, Noguchi R, Tsujinoue H, et al. Tissue inhibitor of metalloproteinases-1 (TIMP-1) inhibits tumor growth and angiogenesis in the TIMP-1 transgenic mouse model. Int J Cancer. 2003;105(3)

33. Waas ET, Wobbes T, Ruers T, Lomme RM, Hendriks T. Circulating gelatinases and tissue inhibitor of metalloproteinase-1 in colorectal cancer metastatic liver disease. Eur J Surg Oncol. 2006 Sep;32(7)

34. Honkavuori M, Talvensaari-Mattila A, Puistola U, Turpeenniemi-Hujanen T, Santala M. High serum TIMP-1 is associated with adverse prognosis in endometrial carcinoma. Anticancer Res. 2008 SepOct;28(5A)

35. Monach PA, Warner RL, Tomasson G, Specks U, Stone JH, Ding L, Fervenza FC, Fessler BJ, Hoffman GS, Iklé D, Kallenberg CG, Krischer J, Langford CA, Mueller M, Seo P, St Clair EW, Spiera R, Tchao N, Ytterberg SR, Johnson KJ, Merkel PA. Serum proteins reflecting inflammation, injury and repair as biomarkers of disease activity in ANCA-associated vasculitis. Ann Rheum Dis. 2013 Aug;72(8)

36. Määtta M, Talvensaari-Mattila A, Turpeenniemi-Hujanen T, Santala M. Matrix metalloproteinase-2 (MMP-2) and -9 (MMP-9) and their tissue inhibitors (TIMP-1 and TIMP-2) in differential diagnosis between low malignant potential (LMP) and malignant ovarian tumours. Anticancer Res. 2007 JulAug;27(4C)

37. Creemers EE, Davis JN, Parkhurst AM, Leenders P, Dowdy KB, Hapke E, Hauet AM, Escobar PG, Cleutjens JP, Smits JF, Daemen MJ. Deficiency of TIMP-1 exacerbates LV remodeling after myocardial infarction in mice. American Journal of Physiology-Heart and Circulatory Physiology. 2003 Jan $1 ; 284(1)$

\section{Tables}

Table 1. 55 angiogenic factors measured by the multiplex angiogenesis array 


\begin{tabular}{|l|l|l|}
\hline Activin A & FGF-7/KGF & PD-ECGF \\
\hline ADAMTS-1 & GDNF & PDGF-AA \\
\hline Angiogenin & GM-CSF & PDGF-AB/PDGF-BB \\
\hline Angiopoietin-1 & HB-EGF & Persephin \\
\hline Angiopoietin-2 & HGF & CXCL4/PF4 \\
\hline Angiostatin/Plasminogen & IGFBP-1 & PIGF \\
\hline Amphiregulin & IGFBP-2 & Prolactin \\
\hline Artemin & IGFBP-3 & Serpin B5/Maspin \\
\hline Tissue Factor/Factor III & IL-1 beta & Serpin E1/PAI-1 \\
\hline CXCL16 & CXCL8/IL-8 & Serpin F1/PEDF \\
\hline DPPIV/CD26 & LAP (TGF-beta 1) & TIMP-1 \\
\hline EGF & Leptin & TIMP-4 \\
\hline EG-VEGF & CCL2/MCP-1 & Thrombospondin-1 \\
\hline Endoglin/CD105 & CCL3/MIP-1 alpha & Thrombospondin-2 \\
\hline Endostatin/Collagen XVIII & MMP-8 & uPA \\
\hline Endothelin-1 & MMP-9 & Vasohibin \\
\hline FGF acidic & NRG1-beta 1 & VEGF \\
\hline FGF basic & Pentraxin 3 & VEGF-C \\
\hline
\end{tabular}


Table 2. Review of the 55 angiogenic factors and their potential significance in angiodysplasia

\begin{tabular}{|c|c|}
\hline $\begin{array}{l}\text { Significance in } \\
\text { gastrointestinal } \\
\text { angiodysplasia }\end{array}$ & Angiopoietin -1, Angiopoietin -2, Endoglin/CD 105, PDGF-BB, VEGF \\
\hline $\begin{array}{l}\text { Significance in } \\
\text { general } \\
\text { gastrointestinal } \\
\text { angiogenesis }\end{array}$ & $\begin{array}{l}\text { ADAMTS-1, Angiogenin, Amphiregulin, Tissue Factor, DPPIV/CD26, EGF, EG-VEGF, Endoglin/CD105, Endostatin/Collagen } \\
\text { XVIII, FGF-acidic, FGF-basic, FGF7/KGF, HB-EGF, HGF, IL-1b, CXCL8, MMP8, MMP9, PD-ECGF, TSP-1, TSP-2 }\end{array}$ \\
\hline $\begin{array}{l}\text { Known to interact } \\
\text { with VEGF }\end{array}$ & $\begin{array}{l}\text { ADAMTS-1, Amphiregulin, TF, EGF, EG-VEGF, Endostatin/Collagen XVIII, Endothelin-1, FGF-acidic, FGF-basic, FGF4, } \\
\text { FGF7/KGF, GM-CSF, HGF, IGFBP-2, IL-1b, CXCL8, CCL2, CCL3, MMP8, MMP9, NRG1-b1, PD-ECGF, PDGF-BB, PIGF, Serpin B5, } \\
\text { Serpin E1, Serpin F1, TSP-1, TSP-2, uPA, Vasohibin, VEGF-C }\end{array}$ \\
\hline $\begin{array}{l}\text { Known to interact } \\
\text { with the } \\
\text { Angiopoietins }\end{array}$ & GM-CSF, NRG1-b1, PDGF-BB \\
\hline
\end{tabular}

Table 3. Results of relative levels of the five significant factors detected by the multiplex analysis in controls and SBA patients 


\begin{tabular}{|c|c|c|c|c|c|}
\hline Column1 & Angiopoietin-1 & Angiopoietin-2 & Endostatin & PDGF-AA & TIMP1 \\
\hline \multicolumn{6}{|l|}{ Controls } \\
\hline C1 & 2049 & 265 & 1602.5 & 3001 & 5508 \\
\hline C2 & 3265.5 & 265 & 2785.5 & 1812.5 & 2274.5 \\
\hline C3 & 2763.5 & 295 & 709.5 & 431 & 2096.5 \\
\hline C4 & 825 & 82 & 695.5 & 1237.5 & 3546 \\
\hline C5 & 1068 & 265.5 & 546 & 1244.5 & 3216.5 \\
\hline C6 & 413.5 & 106.5 & 1057 & 427.5 & 3932.5 \\
\hline C7 & 1375.5 & 152.5 & 740.5 & 823.5 & 3152 \\
\hline Mean & 1680 & 204.5 & 1162.357143 & 1183 & 3389.428571 \\
\hline \multicolumn{6}{|c|}{ SBA patients } \\
\hline SBA1 & 640 & 268 & 535.5 & 583 & 1731 \\
\hline SBA2 & 462.5 & 360 & 385 & 79.5 & 458.5 \\
\hline SBA3 & 818 & 204.5 & 638 & 190.5 & 3164 \\
\hline SBA4 & 512.5 & 374.5 & 495 & 349 & 3188 \\
\hline SBA5 & 986 & 410 & 520 & 843 & 1149.5 \\
\hline SBA6 & 1131 & 459 & 581 & 570.5 & 3003.5 \\
\hline SBA7 & 186.5 & 201 & 125 & 804.5 & 714.5 \\
\hline Mean & 676.6428571 & 325.2857143 & 468.5 & 488.5714286 & 1915.571429 \\
\hline$P$ value & 0.0327 & 0.035 & 0.044 & 0.0473 & 0.0359 \\
\hline $95 \% \mathrm{Cl}$ & $97.32-1909.39$ & $10.02-231.55$ & $21.92-1365.79$ & $11.15-1576.71$ & $114.162-283.55$ \\
\hline
\end{tabular}




\begin{tabular}{|c|c|c|c|}
\hline & $\begin{array}{l}\text { TIMP1 } \\
\text { (ng/mL) }\end{array}$ & $\begin{array}{l}\text { Endostatin } \\
(\mathrm{ng} / \mathrm{mL})\end{array}$ & $\begin{array}{l}\text { PDGF-AA } \\
(\mathrm{ng} / \mathrm{mL})\end{array}$ \\
\hline \multicolumn{4}{|c|}{ SBA Patients } \\
\hline \multicolumn{4}{|l|}{$n=20$} \\
\hline Mean & 425.102 & 556.803 & 36080.105 \\
\hline Median & 392.248 & 524.521 & 32573.18 \\
\hline Range & $134.34-616.3$ & $123.26-909.39$ & $376.08-81945.71$ \\
\hline \multicolumn{4}{|c|}{ Controls } \\
\hline \multicolumn{4}{|l|}{$n=20$} \\
\hline Mean & 558.924 & 387.120 & 37303.636 \\
\hline Median & 520.254 & 320.957 & 377769.09 \\
\hline Range & $374.2-829.94$ & $123.26-925.55$ & $478.37-70024.52$ \\
\hline$p$ value & 0.0028 & 0.0336 & 0.866 \\
\hline $95 \% \mathrm{Cl}$ & $(230.993)-(52.299)$ & $13.885-325.482$ & N/A \\
\hline
\end{tabular}

Table 4. Mean serum levels of TIMP1, Endostatin and PDGF-AA in SBA patients and controls measured by ELISA technique.

Table 5. Results of one way ANOVA testing to determine confounding variables for levels of TIMP1, Endostatin and PDGF-AA in SBA patients and controls 


\begin{tabular}{|c|c|c|c|c|c|}
\hline One way ANOVA & Group & & TIMP1 & Endostatin & PDGF-AA \\
\hline \multirow[t]{4}{*}{ Age $>50$ yrs } & SBA patients & f ratio & $\mathrm{N} / \mathrm{A}$ & $\mathrm{N} / \mathrm{A}$ & $\mathrm{N} / \mathrm{A}$ \\
\hline & & $\mathrm{p}$ value & $\mathrm{N} / \mathrm{A}$ & $\mathrm{N} / \mathrm{A}$ & $\mathrm{N} / \mathrm{A}$ \\
\hline & Controls & f ratio & 1.204 & 4.929 & 0.422 \\
\hline & & $p$ value & 0.288 & 0.04 & 0.524 \\
\hline \multirow[t]{4}{*}{ Gender } & SBA patients & f ratio & 0.066 & 1.172 & 3.398 \\
\hline & & $\mathrm{p}$ value & 0.8 & 0.294 & 0.082 \\
\hline & Controls & f ratio & 1.072 & 0.119 & 1.145 \\
\hline & & $p$ value & 0.315 & 0.734 & 0.299 \\
\hline \multirow[t]{4}{*}{ Anaemia } & SBA patients & f ratio & $\mathrm{N} / \mathrm{A}$ & $\mathrm{N} / \mathrm{A}$ & $\mathrm{N} / \mathrm{A}$ \\
\hline & & $\mathrm{p}$ value & $\mathrm{N} / \mathrm{A}$ & $\mathrm{N} / \mathrm{A}$ & $\mathrm{N} / \mathrm{A}$ \\
\hline & Controls & f ratio & 3.403 & 2.924 & 0.023 \\
\hline & & $p$ value & 0.083 & 0.106 & 0.881 \\
\hline
\end{tabular}

Table 6. Variability in significance of mean levels of TIMP1, Endostatin and PDGF-AA when controlled for variables

\begin{tabular}{|c|c|c|c|c|}
\hline \multicolumn{2}{|l|}{ Factor controlled for } & \multirow{2}{*}{$\begin{array}{l}\text { TIMP1 } \\
\text { ng/mL }\end{array}$} & \multirow{2}{*}{$\begin{array}{l}\text { Endostatin } \\
\mathrm{ng} / \mathrm{mL}\end{array}$} & \multirow{2}{*}{$\begin{array}{l}\text { PDGF-AA } \\
\mathrm{ng} / \mathrm{mL}\end{array}$} \\
\hline & & & & \\
\hline \multirow[t]{4}{*}{ Age $>50$ years } & SBA patients $n=20$ & 425.102 & 556.804 & 36080.105 \\
\hline & Controls $n=7$ & 602.597 & 523.571 & 41706.68 \\
\hline & $\mathrm{p}$ value & 0.0059 & 0.777 & 0.586 \\
\hline & $95 \% \mathrm{Cl}$ & $(298.295)-(56.695)$ & & \\
\hline \multirow[t]{4}{*}{ Gender } & SBA $n=12$ & 418.264 & 525.036 & 31299.176 \\
\hline & Controls $n=9$ & 521.881 & 349.188 & 43397.475 \\
\hline & $p$ value & 0.03 & 0.049 & 0.243 \\
\hline & $95 \% \mathrm{Cl}$ & $11.144-196.089$ & $(415.449)-(2.117)$ & \\
\hline \multirow[t]{4}{*}{ Presence of anaemia } & SBA patients $n=17$ & 410.767 & 525.896 & 37684.034 \\
\hline & Controls $n=6$ & 647.567 & 260.201 & 38514.865 \\
\hline & $\mathrm{p}$ value & 0.003 & 0.019 & 0.464 \\
\hline & $95 \% \mathrm{Cl}$ & $(384.191)-(89.409)$ & $47.553-483.836$ & \\
\hline
\end{tabular}

